\title{
Assessing the relationship between psychosocial stressors and psychiatric resilience among Chilean disaster survivors
}

Cristina A. Fernandez, Karmel W. Choi, Brandon D. L. Marshall, Benjamin Vicente, Sandra Saldivia, Robert Kohn, Karestan C. Koenen, Kristopher L. Arheart and Stephen L. Buka

\section{Background}

According to the stress inoculation hypothesis, successfully navigating life stressors may improve one's ability to cope with subsequent stressors, thereby increasing psychiatric resilience.

\begin{abstract}
Aims
Among individuals with no baseline history of post-traumatic stress disorder (PTSD) and/or major depressive disorder (MDD) to determine whether a history of a stressful life event protected participants against the development of PTSD and/or MDD after a natural disaster.
\end{abstract}

\begin{abstract}
Method
Analyses utilised data from a multiwave, prospective cohort study of adult Chilean primary care attendees (years 2003-2011; $n=1160$ ). At baseline, participants completed the composite International Diagnostic Interview (CIDI), a comprehensive psychiatric diagnostic instrument, and the List of Threatening Experiences, a 12-item questionnaire that measures major stressful life events. During the study (2010), the sixth most powerful earthquake on record struck Chile. One year later (2011), the CIDI was re-administered to assess post-disaster PTSD and/or MDD.
\end{abstract}

\section{Results}

Marginal structural logistic regressions indicated that for every one-unit increase in the number of pre-disaster stressors, the odds of developing post-disaster PTSD or MDD increased $(\mathrm{OR}=1.21,95 \% \mathrm{Cl} 1.08-1.37$, and $\mathrm{OR}=1.16,95 \% \mathrm{Cl} 1.06-1.27$ respectively). When categorising pre-disaster stressors, individuals with four or more stressors (compared with no stressors) had higher odds of developing post-disaster PTSD $(\mathrm{OR}=2.77$, 95\% Cl 1.52-5.04), and a dose-response relationship between pre-disaster stressors and post-disaster MDD was found.

\section{Conclusions}

In contrast to the stress inoculation hypothesis, results indicated that experiencing multiple stressors increased the vulnerability to developing PTSD and/or MDD after a natural disaster. Increased knowledge regarding the individual variations of these disorders is essential to inform targeted mental health interventions after a natural disaster, especially in under-studied populations.

\section{Keywords}

Post-traumatic stress disorder; low- and middle-income countries; epidemiology; depressive disorders; natural disasters.

\section{Copyright and usage}

(c) The Author(s), 2020. Published by Cambridge University Press on behalf of the Royal College of Psychiatrists.
Many find inspiration and meaning in Nietzsche's famous words, 'What does not destroy me, makes me stronger'. The theory behind these words is the stress inoculation hypothesis, which attempts to predict an individual's reaction to a stressful event on the basis of their past experiences. ${ }^{1}$ Specifically, this hypothesis posits that experiencing manageable stressors may improve an individual's ability to cope with future stressors by providing a context in which to practise effective coping skills and build a sense of mastery over stressors. ${ }^{2}$ This in turn could enhance resilience broadly defined as positive psychological adaptation to adversity ${ }^{3}-$ and reduce later vulnerability to poor mental health outcomes. ${ }^{1,4}$ However, whether this holds true when individuals are later exposed to traumatic stressors, specifically for some of the most common and debilitating stress-related clinical conditions such as major depressive disorder (MDD) or post-traumatic stress disorder (PTSD), remains open to debate. ${ }^{5,6}$

\section{Manageable versus traumatic stressors}

Traumatic stressors are different from manageable stressors in that manageable stressors are typically less severe, allowing most individuals to engage in coping efforts without exceeding their capacity to manage such stressors. For example, someone who has lost their job may find certain strategies (e.g. problem-solving, physical exercise and social support) helpful in managing the stress of unemployment; in turn, this experience could provide a template for coping effectively with later stressors. If the later stressor were, say, another episode of unemployment, this would be an example of direct tolerance - a type of inoculation where the prior stressor is the same as the later exposure. ${ }^{3}$ Conversely, if the later stressor were a divorce, this would be an example of cross tolerance - a type of inoculation where the prior stressor is different from the later exposure. As illustrated in these examples, both prior and later exposures are manageable stressors. According to the inoculation hypothesis, this would increase the likelihood of successful initial coping and subsequent inoculation against stress-related disorders such as MDD and/or PTSD.

Compared with manageable stressors, traumatic stressors (e.g. rape, combat) are more extreme in nature and can overwhelm an individual's ability to cope effectively by inducing emotional distress that exceeds what they can independently manage and/or exhausts the capacity of the stress response system. ${ }^{7}$ Literature suggests that prior stressors, particularly those that are traumatic and unmanageable, can increase risk for later psychiatric disorders such as PTSD and/or MDD. ${ }^{8}$ For example, individuals with maladaptive cognitive vulnerabilities (e.g. negative attentional bias) developed in response to earlier stressors may more readily develop future psychiatric disorders $^{9}$ - as consistent with a stress sensitisation model. This model is similar to the concepts of 'kindling' and/or 'weathering', in which earlier vulnerability to psychopathology triggered by initial stressful 
experiences is posited to decrease the threshold of stress exposure required for developing subsequent psychopathology. ${ }^{10}$ However, previous work has also shown that prior trauma exposure alone does not predict later PTSD, except among those who developed PTSD after the prior exposure. ${ }^{11,12}$ If someone has experienced a prior stressor but did not develop a psychiatric disorder such as PTSD and/or MDD, this suggests that they successfully managed this stressor from a psychological perspective (e.g. seeking support, establishing daily routines, finding meaning) and may thus be prepared to cope successfully with future traumatic exposures - as consistent with the inoculation hypothesis. However, these hypotheses require further investigation in trauma-exposed populations.

\section{The PREDICT study, 2010 earthquake/tsunami and hypotheses of the current study}

The main objective of the current study was to test the inoculation hypothesis in an understudied Chilean population. In the midst of a multiwave, cross-national cohort study, the PREDICT study, one of the most powerful earthquakes on record, measuring 8.8 on the Richter scale, struck the coast of central Chile (27 February 2010). This disaster resulted in over 500 deaths, 12000 injured, 800000 displaced, and hundreds of thousands of buildings damaged or destroyed. ${ }^{13}$ The cities of Concepción and Talcahuano, where this cohort was based, were major urban areas that experienced most damage from the earthquake and its subsequent effects, including a tsunami that hit Talcahuano. ${ }^{13}$ Chile is particularly vulnerable to earthquakes and tsunamis owing to the country's geographic location on an arc of volcanos and fault-lines circling the Pacific Ocean, otherwise known as the Ring of Fire. Individuals in these high-risk locations are often exposed to recurrent disasters and are therefore likely to be at higher risk for developing post-disaster psychological problems such as PTSD and/or MDD. ${ }^{14}$

\section{Hypotheses of the current study}

We sought to assess, among individuals with no pre-disaster psychiatric history of MDD or PTSD, whether a history of prior stressors was associated with psychiatric resilience, as evidenced by the absence of negative outcomes where otherwise expected - specifically, whether it protected against the post-disaster development of PTSD and/or MDD, two of the most common psychiatric reactions following disasters. ${ }^{14}$ The three hypotheses are: (a) prior experience of a natural disaster will protect against developing post-disaster PTSD and/or MDD (i.e. direct tolerance); (b) prior experience of manageable stressors will protect against developing post-disaster PTSD and/or MDD (i.e. cross tolerance); and (c) there will be a dose-response negative relationship between the number of prior stressors and increased odds of post-disaster PTSD and/or MDD. This study will provide an unprecedented opportunity to answer these questions in an international setting, providing culturally and context-specific information about the risk factors associated with developing psychopathology after a disaster.

\section{Method}

\section{Participants}

The current analysis utilises two waves of data from the Chilean site of the PREDICT study $(n=1160)$, a cross-national prospective cohort study with the primary aim of predicting mental health outcomes in primary care attendees. ${ }^{15-17}$ Participants over the age of 15 were recruited from 10 primary care centres within the national

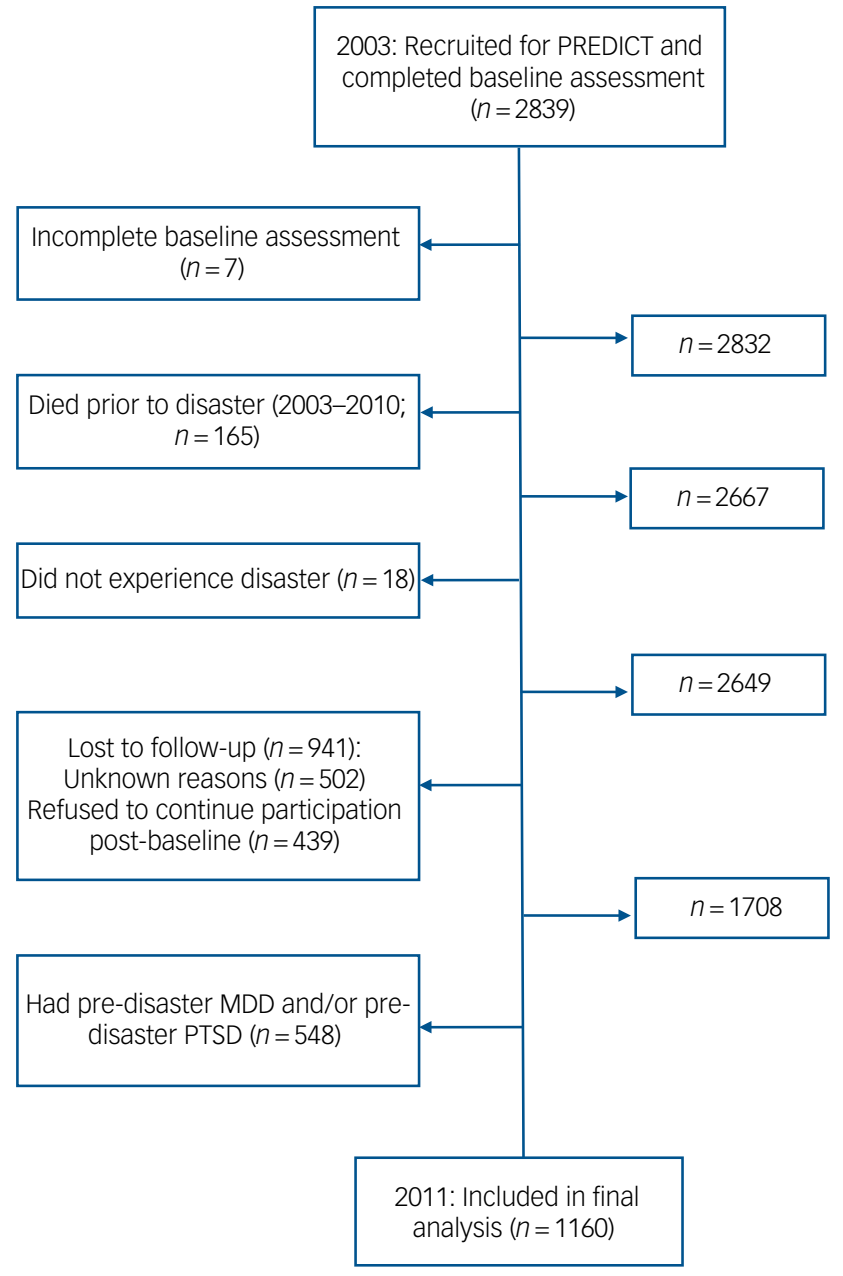

Fig. 1 Flow diagram of excluded/ineligible individuals: The PREDICT study (2003-2011).

MDD, major depressive disorder; PTSD, post-traumatic stress disorder.

healthcare service (used by approximately $75 \%$ of the population) in the cities of Concepción and Talcahuano, Chile. ${ }^{15}$ Of the 3000 participants who initially agreed to take part, 2839 completed the baseline pre-disaster assessment in 2003 and 1708 completed the postdisaster assessment in 2011, 1 year after the disaster occurred. ${ }^{18}$ Because the inoculation hypothesis assumes that individuals have successfully coped with prior stressors (i.e. not developing PTSD and/or MDD), those with a pre-disaster MDD and/or PTSD diagnosis (according to the baseline Composite International Diagnostic Interview (CIDI) scores) were excluded. The exclusion and inclusion criterion used to obtain the analytic sample $(n=1160)$ are shown in Fig. 1.

\section{Ethics statement}

Written informed consent was obtained from all participants. The authors assert that all procedures contributing to this work comply with the ethical standards of the relevant national and institutional committees on human experimentation and with the Helsinki Declaration of 1975, as revised in 2008. All procedures involving human participants/patients were approved by the Institutional Review Board at the University of Concepción. The current study utilises secondary analysis using de-identified data; therefore, institutional review board approval was not necessary. 


\section{Measurements}

Dependent variables: post-disaster MDD and post-disaster PTSD (as measured in 2011)

The Composite International Diagnostic Interview (CIDI), Spanish version $2.1^{19}$ was used to assess pre- and post-disaster MDD and PTSD. The CIDI is a fully structured psychiatric diagnostic instrument that assesses psychiatric disorders via computerised algorithms according to DSM-IV-TR criteria. ${ }^{20}$ The CIDI has good psychometric properties, with excellent interrater reliability, good test-retest reliability and good validity ${ }^{21}$ and is used widely throughout the world. ${ }^{22}$ The CIDI is administered by lay interviewers and does not use outside informants or medical records. ${ }^{19}$ It also uses skip patterns to efficiently diagnose the presence/absence of a disorder, although this yields limited systematic symptom-level information and may reduce power by excluding individuals with subclinical diagnoses. The translated version of the CIDI is an official World Health Organization Spanish version ${ }^{23,24}$ and has been validated in prior national studies conducted in Chile. A prior validation study found that each CIDI section showed moderate to excellent kappa estimates. ${ }^{25}$

The Depressive Disorders module (section E) of the CIDI was used to diagnose post-disaster MDD in the past 12 months (i.e. since the 2010 disaster occurred). These questions follow the DSM-IV-TR symptom criteria. A full description of this module can be found in supplementary Appendix 2, available at https:// doi.org/10.1192/bjp.2020.88. In addition to the post-disaster MDD assessment, a modified version of the PTSD module of the CIDI (section F) Spanish version $2.1^{19}$ was used to assess post-disaster PTSD. This module asks about all 21 PTSD symptoms from the DSM-IV-TR. ${ }^{26}$ It is important to note the details of the customisations to the post-disaster PTSD module. There are two major differences in the original PTSD module of the CIDI compared with the modified version. First, unlike the original PTSD module, the modified version does not begin the interview with a complete enumeration of potentially traumatic events as operationalised by the DSM-IV-TR. Instead, participants were only asked whether they had or had not experienced the 2010 natural disaster (i.e. criterion A.1). If the participant did not endorse being in the 2010 disaster, they were screened out of the study. No other history of potentially traumatic events was assessed, to ensure that the assessment was measuring PTSD from the 2010 disaster only (i.e. only individuals with disaster-related PTSD were captured). ${ }^{18}$

The second major difference in the modified PTSD module was that all the PTSD symptoms were anchored to assess PTSD symptoms due to the 2010 disaster only. This required minor modifications to all the questions, so that they referred directly to it. For example, a question used to measure avoidance symptoms reads: 'Were you trying to force yourself to not think or talk about the earthquake/tsunami?'; and a question used to measure a symptom of re-experiencing is: 'After the earthquake/tsunami, did you have nightmares?'. Note that all the assessment questions referenced the 2010 disaster when asking about PTSD symptoms. ${ }^{18}$

\section{Independent variables: pre-disaster stressors (as measured in 2003)}

\section{List of Threatening Experiences: The List of Threatening} Experiences (LTE) is a 12-item dichotomous response questionnaire used to measure major categories of stressful life events (from the previous 6 months) involving moderate or long-term threat. ${ }^{27}$ Stressful events include: serious illness, injury or assault to self or close relative; death of parent, child, spouse/partner, close family friend or another relative (e.g. aunt, cousin, grandparent); marital or relationship separation; serious problem with a close friend, neighbour or relative; unemployment; recent job termination; major financial crisis; problems with the police (including a court appearance); and something of value lost or stolen. ${ }^{27}$ At the baseline assessment, participants indicated whether each of the 12 different stressful life events had occurred in the previous 6 months. The total score is the sum of the individual items (maximum score: 12). ${ }^{28}$ The LTE has been shown to have good psychometric properties, with excellent test-retest reliability, good interrater reliability and high concurrent validity. ${ }^{27}$ To examine dose-response relationships between the number of pre-disaster stressors and risk of post-disaster PTSD and/or MDD, the total score was categorised $(0,1,2,3, \geq 4$ stressors) on the basis of the distribution of the sample. Stressors captured by the LTE were conceptualised as 'cross tolerance' for the current study.

Prior disaster experience: In the CIDI baseline assessment, participants indicated whether they had previously experienced a natural disaster (i.e. any natural disaster prior to the 2010 earthquake/tsunami) as part of the 'potentially traumatic events' portion of the PTSD module of the CIDI. The disaster stressor captured was conceptualised as 'direct tolerance' for the current study.

\section{Confounding variables}

Confounding variables were selected on the basis of the literature regarding known risk factors for pre-disaster stressors and post-disaster PTSD and MDD. ${ }^{29-31}$ These include age, gender and educational attainment. Age was collapsed into 'middle-aged' or 'not middle-aged' (i.e. 45-55 years old versus any other age), because subsequent post hoc analyses showed significant differences only between these two age groups. Education was collapsed into 'illiterate/elementary school' versus 'high school/college' for the same reason. ${ }^{18}$

\section{Statistical analyses}

\section{Loss to follow-up}

As described in our previous work with these data, ${ }^{18}$ there is potential for selection bias due to differential loss to follow-up in this longitudinal study design. To examine this possibility, $\chi^{2}$ and multivariable logistic regression analyses were conducted to examine the characteristics of those who were lost to follow-up ( $n=941$ (33.1\% of the original sample); Fig. 1). The 'lost to follow-up' category included individuals who refused subsequent assessments post-baseline, died, or were lost to follow-up for unknown reasons. Among these 941 individuals, there were significantly more females than males (69\% v. 30\%; $\left.\chi^{2}=14.84, P<0.001\right)$, more participants with a high-school/college education compared with those who were illiterate or had an elementary school education $\left(73.4 \%\right.$ v. $\left.26.6 \% ; \chi^{2}=11.89, P=0.001\right)$, and more individuals who were not middle-aged relative to those who were middleaged $\left(83.5 \%\right.$ v. $\left.16.5 \% ; \chi^{2}=4.71, P=0.03\right)$. Multivariable logistic regression models predicting loss to follow-up replicated these findings (results not shown). ${ }^{18}$ Additional sensitivity analyses examining the differences in the rate of other pre-disaster disorders among those who were and were not lost to follow-up are displayed in supplementary Appendix 1.

\section{Inverse probability weighting}

To mitigate the potential selection bias due to differential loss to follow-up, inverse probability censoring weights were calculated. To estimate the censoring weights, the predicted probability of not dropping out based on each participant's exposure (i.e. pre-disaster stressors) and confounder values (i.e. gender, age and education) were estimated using a multivariable logistic regression model. Weights were calculated for each participant as the inverse of this probability. Thus, the weights can be described as the number of participants who are like individual $i$ (in terms of their 
exposure and confounder values) who would have been in the risk set at the post-disaster assessment in the absence of drop out. In sum, the inverse probability censoring weights create a pseudopopulation had drop out been random (with respect to exposure and confounder values). Weights were stabilised to preserve the amount of information in the observed data and minimise variability. ${ }^{18,32}$

To mitigate potential confounding bias, inverse probability exposure weights were calculated. To estimate the exposure weights, the probability of each individual's exposure (i.e. pre-disaster stressors) given their confounder values (i.e. gender, age and education) was modelled. The final weights can be described as a pseudo-population where each participant's exposure is independent of their measured confounders. ${ }^{33}$ Weights were stabilised to preserve the amount of information in the observed data and minimise variability. ${ }^{18,32}$

\section{List of threatening experiences - questions about assault}

It is worth noting that two of the LTE questions ask about assault to self $(n=204 ; 17.6 \%)$ or assault to others $(n=254 ; 21.9 \%)$. According to the DSM-IV-TR criteria, these experiences are considered potentially traumatic events and should not be considered as 'manageable stressors'. Therefore, all analyses were conducted two ways: one set of analyses with all 12 of the items of the LTE (i.e. those presented in final tables) and another set of analyses with the two items about assault excluded. Results from both sets of analyses were not statistically different from each other (i.e. the confidence intervals overlapped and the point estimates only marginally changed). The similarity of results is likely due to the analytic sample used to operationalise 'manageable stressors'. Further, given that the LTE has been validated as is (the full 12-item questionnaire), the questions regarding assault were kept for all analyses and we will continue to use the term 'manageable stressors' for continuity purposes and to accurately reflect the wording of the inoculation hypothesis.

\section{Post hoc sensitivity analyses}

It is important to note that post hoc sensitivity analyses excluding individuals with other pre-disaster disorders besides PTSD and MDD (e.g. alcohol misuse, anxiety disorders, dysthymia, nonaffective psychotic disorders) did not substantially change the findings. Further, there were no significant interactions (either additive or multiplicatative) between prior disaster experience (before the 2010 earthquake/tsunami) and stressors. Our results are robust and the effect estimates did not vary substantially, these findings are available on request.

\section{Analysis plan}

As mentioned above, because the inoculation hypothesis assumes that individuals effectively manage their stressors to cope with subsequent adversity, those with a pre-disaster MDD or pre-disaster PTSD (according to the CIDI at the baseline assessment) were excluded.

The study hypotheses utilised a series of marginal structural logistic models, with exposure and confounding inverse probability weights. To test direct tolerance, we examined whether a history of being in a disaster (prior to the 2010 disaster) protected against developing post-disaster PTSD and MDD. To test cross-tolerance, we examined whether a history of non-disaster stressors (i.e. total LTE score) protected against developing post-disaster PTSD and MDD. STATA/MP version 12 for Macintosh was used for data management and statistical analyses. ${ }^{34}$

\section{Results}

\section{Descriptive information}

Among the individuals with post-disaster PTSD ( $n=106$; Table 1$)$ and post-disaster MDD ( $n=167$; Table 2$)$, most were female, not middle-aged, had a high-school/college education and had not experienced a disaster prior to the 2010 earthquake/tsunami. The distribution of the LTE scores in all subsamples was positively skewed.

\section{Marginal structural logistic regression models - PTSD}

As shown in Table 3, model 1 tested the risk of PTSD associated with direct tolerance (i.e. prior disaster experience). Models 2 and 3 tested the risk of PTSD associated with cross tolerance (i.e. prior non-disaster stressor experience). Prior disaster exposure was not a significant predictor of post-disaster PTSD. On the other hand, for every unit increase in prior non-disaster stressors, the odds of developing post-disaster PTSD increased $(\mathrm{OR}=1.21,95 \%$ CI 1.08-1.37; $P=0.001 ;$ model 2). When these stressors were categorised, those who experienced $\geq 4$ stressors (versus 0 stressors) had increased odds of developing post-disaster PTSD $(\mathrm{OR}=2.77$, 95\% CI 1.52-5.04; $P=0.001$; model 3).

\section{Marginal structural logistic regression models - MDD}

As displayed in Table 4, model 1 tested the risk of MDD associated with prior disaster experience. Models 2 and 3 tested the risk of MDD associated with prior non-disaster stressor experiences. Prior disaster exposure was not a significant predictor of post-disaster MDD (model 1). For every 1-unit increase in prior non-disaster stressors, the odds of developing post-disaster MDD increased (OR $=1.16,95 \%$ CI 1.06-1.27; $P=0.001$; model 2). When stressors were categorised, experiencing any number of stressors (relative to 0 stressors) significantly increased the odds of developing post-disaster MDD in a dose-response fashion (model 3).

\section{Discussion}

The current study tested the applicability of the inoculation hypothesis on psychiatric vulnerability in an understudied international population who had experienced a natural disaster. To do so, we assessed whether a history of stressful life events among Chilean adults with no lifetime history of PTSD and/or MDD decreased the odds that a subsequent traumatic experience (i.e. exposure to an earthquake/tsunami) would trigger MDD and/or PTSD. Cumulatively, the findings did not support direct or indirect inoculation. In fact, the results were in the opposite hypothesised direction and are therefore reflective of the stress sensitisation model, which states that experiencing multiple stressors increases the probability of developing a psychiatric disorder (as opposed to more resilience, implied in the inoculation hypothesis).

Because a history of pre-disaster stressors increased the risk of developing post-disaster PTSD and/or MDD, it is likely that this Chilean sample experienced 'stress sensitisation'. Stress sensitisation posits that a stressor will make an individual more vulnerable to the negative effects of subsequent stressors, rather than developing resilience. ${ }^{35-37}$ Therefore, an individual who has experienced several stressors in their lifetime will be at higher risk for developing a psychiatric disorder. ${ }^{36}$ This theory is supported by substantial literature ${ }^{11,12}$ and has also been used to explain individual differences in the development, 


\begin{tabular}{|c|c|c|c|}
\hline & $\begin{array}{l}\text { Total sample } \\
(n=1160), n\end{array}$ & $\begin{array}{l}\text { Participants with post-disaster } \\
\text { PTSD }(n=106 ; 9.1 \%), n(\%)\end{array}$ & $\begin{array}{l}\text { Participants without post- } \\
\text { disaster PTSD ( } n=1054 \text {; } \\
90.9 \%), n(\%)\end{array}$ \\
\hline \multicolumn{4}{|l|}{ Gender } \\
\hline Male & 348 & $19(5.5)$ & $329(94.5)$ \\
\hline Female & 812 & $87(10.7)$ & 725 (89.3) \\
\hline \multicolumn{4}{|l|}{ Age, years } \\
\hline 45-54 & 205 & $29(14.2)$ & $176(85.9)$ \\
\hline$<45$ and $55+$ & 955 & $77(8.1)$ & 878 (91.9) \\
\hline \multicolumn{4}{|l|}{ Education } \\
\hline High school/college & 783 & $62(7.9)$ & $721(92.1)$ \\
\hline Illiterate/elementary school & 375 & $44(11.7)$ & $331(88.3)$ \\
\hline Missing data & 2 & 0 & $2(100)$ \\
\hline \multicolumn{4}{|l|}{ Post-disaster MDD } \\
\hline Yes & 167 & $38(22.8)$ & $129(77.3)$ \\
\hline No & 993 & $68(6.9)$ & $925(93.2)$ \\
\hline \multicolumn{4}{|l|}{ Pre-disaster stressors } \\
\hline \multicolumn{4}{|l|}{ Continuous LTE total score ${ }^{a}$} \\
\hline 0 & 420 & $32(7.6)$ & $388(92.4)$ \\
\hline 1 & 330 & $27(8.2)$ & $303(91.8)$ \\
\hline 2 & 174 & $17(9.8)$ & $157(90.2)$ \\
\hline 3 & 125 & $9(7.2)$ & $116(92.8)$ \\
\hline 4 & 55 & $10(18.2)$ & $45(81.8)$ \\
\hline 5 & 28 & $3(10.7)$ & $25(89.3)$ \\
\hline 6 & 17 & $3(17.7)$ & $14(82.4)$ \\
\hline 7 & 7 & $3(42.9)$ & $4(57.1)$ \\
\hline 8 & 2 & $1(50.0)$ & $1(50.0)$ \\
\hline 9 & 1 & 0 & $1(100.0)$ \\
\hline 10 & 1 & $1(100.0)$ & 0 \\
\hline \multicolumn{4}{|l|}{ Categorised LTE total score } \\
\hline 0 & 420 & $32(7.6)$ & $388(92.4)$ \\
\hline 1 & 330 & $27(8.2)$ & $303(91.8)$ \\
\hline 2 & 174 & $17(9.8)$ & $157(90.2)$ \\
\hline 3 & 125 & $9(7.2)$ & $116(92.8)$ \\
\hline$\geq 4$ & 111 & $21(18.9)$ & $90(81.1)$ \\
\hline \multicolumn{4}{|l|}{ Disaster ${ }^{\mathrm{b}}$} \\
\hline Yes & 230 & $22(9.6)$ & $208(90.4)$ \\
\hline No & 926 & $84(9.1)$ & $842(90.9)$ \\
\hline Missing data & 4 & 0 & $4(100.0)$ \\
\hline
\end{tabular}

recurrence and maintenance of psychiatric disorders such as PTSD and/or MDD. ${ }^{38,39}$

Unfortunately, the majority of research on PTSD has investigated risk associated with prior traumatic stressors, not manageable stressors. ${ }^{29}$ This is especially true in populations outside of the USA. In the current study, results indicated that for every one-unit increase in pre-disaster stressors, the odds of developing post-disaster PTSD increased by $21 \%$. However, when stressors were categorised, only participants who experienced $\geq 4$ stressors (i.e. the highest category), relative to 0 stressors, had higher odds of developing post-disaster PTSD. This suggests that the number of prior manageable stressors needs to cross a severity threshold ( $\geq 4$ stressors) to affect future vulnerability to PTSD. ${ }^{40}$

In contrast to the PTSD literature, it is well-known that 'nontraumatic' psychosocial stressors play an essential role in the etiology of MDD. ${ }^{9,10}$ Other literature in international high-trauma settings has suggested that manageable stressors can actually have a stronger impact than traumatic stressors on mental health outcomes. ${ }^{41,42}$ These adversities may leave residual vulnerabilities in the individual, thus increasing the probability of developing MDD because of sensitisation. ${ }^{10}$ Furthermore, cumulative adversity tends to be more harmful than a single episode, owing to the depletion of coping resources over time. ${ }^{37,43}$ This conceptualisation reflects the results of the current study, which indicated a dose-response relationship between the number of pre-disaster stressors and the risk of post- disaster MDD. These stressful events may be associated with depres$\operatorname{sion}^{38}$ through behavioural (e.g. poor coping mechanisms), cognitive (e.g. negative attention biases, rumination ${ }^{9,44}$ ) and/or biological mechanisms (e.g. physiological stress response dysregulation ${ }^{37,39,45}$ ). Although these pathways were not included in the present study, they merit additional investigation in future longitudinal studies.

\section{Strengths and limitations}

The present study has some limitations worth noting. First, there is potential for measurement error of the exposure variables (i.e. predisaster stressors and pre-disaster PTSD and MDD). The primary concern is that the baseline examination was administered 7 years before the disaster (2003). New stressors that occurred between the baseline assessment and the earthquake (i.e. between 2003 and 2010) may have been missed. It is also possible that individuals developed pre-disaster PTSD and/or pre-disaster MDD during this 7 -year period. Given that our analytic sample excluded individuals with any history of pre-disaster PTSD and/or MDD, those who had PTSD and/or MDD would be categorised as false negatives, leading to biased results.

Similarly, there is potential for misclassification of the outcome variables (post-disaster PTSD and/or post-disaster MDD). Given that the post-disaster PTSD assessment was administered approximately a year after the disaster occurred (2011), there are some 


\begin{tabular}{|c|c|c|c|}
\hline & $\begin{array}{l}\text { Total sample } \\
(n=1160), n\end{array}$ & $\begin{array}{l}\text { Participants with post-disaster } \\
\text { MDD }(n=167 ; 14.4 \%), n(\%)\end{array}$ & $\begin{array}{l}\text { Participants without post-disaster } \\
\operatorname{MDD}(n=993 ; 85.6 \%), n(\%)\end{array}$ \\
\hline \multicolumn{4}{|l|}{ Gender } \\
\hline Male & 348 & $25(7.2)$ & $323(92.8)$ \\
\hline Female & 812 & $142(17.5)$ & $670(82.5)$ \\
\hline \multicolumn{4}{|l|}{ Age, years } \\
\hline $45-54$ & 205 & 40 (19.5) & $165(80.5)$ \\
\hline$<45$ and $55+$ & 955 & $127(13.3)$ & $828(86.7)$ \\
\hline \multicolumn{4}{|l|}{ Education } \\
\hline High school/college & 783 & $115(14.7)$ & $668(85.3)$ \\
\hline Illiterate/elementary school & 375 & 52 (13.9) & $323(86.1)$ \\
\hline Missing data & 2 & 0 & $2(100)$ \\
\hline \multicolumn{4}{|l|}{ Post-disaster PTSD } \\
\hline Yes & 106 & $38(35.9)$ & $68(64.2)$ \\
\hline No & 1054 & $129(12.2)$ & $925(87.8)$ \\
\hline \multicolumn{4}{|l|}{ Pre-disaster stressors } \\
\hline \multicolumn{4}{|l|}{ Continuous LTE total score ${ }^{a}$} \\
\hline 0 & 420 & $39(9.3)$ & $381(90.7)$ \\
\hline 1 & 330 & $53(16.1)$ & $277(83.9)$ \\
\hline 2 & 174 & $32(18.4)$ & $142(81.6)$ \\
\hline 3 & 125 & $22(17.6)$ & $103(82.4)$ \\
\hline 4 & 55 & $8(14.6)$ & 47 (85.5) \\
\hline 5 & 28 & $8(28.6)$ & $20(71.4)$ \\
\hline 6 & 17 & $3(17.7)$ & $14(82.4)$ \\
\hline 7 & 7 & $2(28.6)$ & $5(71.4)$ \\
\hline 8 & 2 & 0 & $2(100)$ \\
\hline 9 & 1 & 0 & $1(100)$ \\
\hline 10 & 1 & 0 & $1(100)$ \\
\hline \multicolumn{4}{|l|}{ Categorised LTE total score } \\
\hline 0 & 420 & $39(9.3)$ & $381(90.7)$ \\
\hline 1 & 330 & $53(16.1)$ & $277(83.9)$ \\
\hline 2 & 174 & $32(18.4)$ & $142(81.6)$ \\
\hline 3 & 125 & $22(17.6)$ & $103(82.4)$ \\
\hline$\geq 4$ & 111 & $21(18.9)$ & $90(81.1)$ \\
\hline \multicolumn{4}{|l|}{ Disaster $^{\mathrm{b}}$} \\
\hline Yes & 230 & $29(12.6)$ & $201(87.4)$ \\
\hline No & 926 & $138(14.9)$ & 788 (85.1) \\
\hline Missing data & 4 & 0 & $4(100)$ \\
\hline
\end{tabular}

\begin{tabular}{|c|c|c|c|c|c|c|c|c|c|}
\hline \multirow[b]{2}{*}{ Independent variable } & \multicolumn{3}{|c|}{ Model 1} & \multicolumn{3}{|c|}{ Model 2} & \multicolumn{3}{|c|}{ Model 3} \\
\hline & OR & $95 \% \mathrm{Cl}$ & $P$ & OR & $95 \% \mathrm{Cl}$ & $P$ & OR & $95 \% \mathrm{Cl}$ & $P$ \\
\hline Disaster ${ }^{\mathrm{b}}$ & 1.06 & $0.65-1.74$ & 0.815 & & & & & & \\
\hline LTE sum: linear & & & & 1.21 & $1.08-1.37$ & 0.001 & & & \\
\hline \multicolumn{10}{|l|}{ LTE sum: categorised } \\
\hline 0 & & & & & & & Ref. & & \\
\hline 1 & & & & & & & 1.07 & $0.63-1.83$ & 0.798 \\
\hline 2 & & & & & & & 1.29 & $0.69-2.39$ & 0.422 \\
\hline 3 & & & & & & & 0.93 & $0.43-2.01$ & 0.856 \\
\hline$\geq 4$ & & & & & & & 2.77 & $1.52-5.04$ & 0.001 \\
\hline
\end{tabular}

individuals who may have had disaster-related PTSD but their symptoms had resolved before the assessment took place. Conversely, individuals may have developed delayed-onset PTSD years after the disaster; these individuals would not have been captured as having disaster-related PTSD in our assessment. Fortunately, misclassification of post-disaster MDD is less likely given that the CIDI only assessed post-disaster MDD from the previous year (i.e. between the disaster occurrence and assessment). Another limitation is that the CIDI automatically generates dichotomised diagnoses because of its skip patterns; therefore, we were unable to examine participants with subclinical PTSD/MDD or to accurately measure PTSD/MDD symptoms. ${ }^{19}$ Excluding those with subclinical diagnoses may also have resulted in a loss of power. Future studies should use multiple time points before and after a disaster to more accurately examine the longitudinal course of PTSD and MDD.

Although the study was conducted in a longitudinal and prospective fashion, the results likely do not reflect causal relationships due to random error, potential type II error, unmeasured confounding, moderators or mediators. For example, the stressor 
Table 4 Marginal structural logistic regression analyses ${ }^{a}$ of pre-disaster stressors predicting post-disaster MDD: The PREDICT study, 2003-2011 $(n=1154)$

\begin{tabular}{|c|c|c|c|c|c|c|c|c|c|}
\hline \multirow[b]{2}{*}{ Independent Variable } & \multicolumn{3}{|c|}{ Model 1} & \multicolumn{3}{|c|}{ Model 2} & \multicolumn{3}{|c|}{ Model 3} \\
\hline & OR & $95 \% \mathrm{Cl}$ & $P$ & OR & $95 \% \mathrm{Cl}$ & $P$ & OR & $95 \% \mathrm{Cl}$ & $P$ \\
\hline Disaster $^{\mathrm{b}}$ & 0.82 & $0.53-1.26$ & 0.358 & & & & & & \\
\hline LTE sum: linear & & & & 1.16 & $1.06-1.27$ & 0.001 & & & \\
\hline \multicolumn{10}{|l|}{ LTE sum: categorised } \\
\hline 0 & & & & & & & Ref. & & \\
\hline 1 & & & & & & & 1.86 & $1.20-2.89$ & 0.006 \\
\hline 2 & & & & & & & 2.19 & $1.31-3.62$ & 0.003 \\
\hline 3 & & & & & & & 2.09 & $1.19-3.69$ & 0.011 \\
\hline$\geq 4$ & & & & & & & 2.27 & $1.27-4.04$ & 0.006 \\
\hline
\end{tabular}

questionnaire did not measure the individual's appraisal of the stressor, their coping response, the stressor's contextual meaning, the frequency/load of the stressful events and/or whether the person achieved complete mastery over the stressor. ${ }^{45}$ This information is pertinent to providing stronger evidence for the inoculation hypothesis - we strongly recommend that future studies examining stress response include these indicators. ${ }^{46}$ Further, the LTE measured stressors from the 6 months prior to the baseline assessment. This is likely not a large enough time frame to capture the extent to which people may have experienced life stressors, which may have led to biased results. Finally, findings may not necessarily generalise to other populations outside of Chile.

Despite these limitations, the current study has many unique strengths. Analyses took advantage of a rare opportunity to study adults who had undergone a psychiatric and stressor evaluation in a large sample prior to exposure to one of the most powerful earthquakes in history, thus providing a clearer understanding of the preexisting risk factors for developing PTSD and/or MDD. ${ }^{18}$ This type of rich longitudinal data does not exist in the disaster literature. ${ }^{14}$ Previous studies that have attempted to address these issues have been severely limited by small convenience samples, lack of diagnostic instruments and scarcity of pre-disaster information. ${ }^{2,18}$ The current study overcomes these limitations and allows for testing of hypotheses not previously possible using a methodologically robust study design. This information is critical to understanding variations in risk of PTSD and/or MDD, with the overall goal of identifying those who may need mental health treatment after a disaster. $^{18}$ By examining who has truly new-onset PTSD and MDD after a natural disaster, the causal mechanisms of these illnesses can be more accurately determined. Increased knowledge regarding the individual variations of these disorders is essential to inform targeted mental health interventions after a natural disaster, especially in under-studied populations. ${ }^{13}$

Cristina A. Fernandez (D) PhD, MSEd, Department of Epidemiology, Harvard T.H. Chan School of Public Health, Boston, MA and Department of Epidemiology, Brown School of Public Health, Providence, RI, USA; Karmel W. Choi, PhD, Department of Epidemiology, Harvard T.H. Chan School of Public Health, Boston, MA, USA; Brandon D.L. Marshall PhD, Department of Epidemiology, Brown School of Public Health, Providence, RI, USA; Benjamin Vicente, MD, PhD, Departamento de Psiquiatría y Salud Mental, Universidad de Concepción, Concepción, Chile; Sandra Saldivia, PhD, Departamento de Psiquiatría yalud Mental, Universidad de Concepción, Concepción, Chile; Robert Kohn (O), MD, Serer University, Providence RI, USA; Karestan C. Koenen, PhD, Department of Epidemiology ، Harvard T.H. Chan School of Public Health, Boston, MA, USA; Kristopher L. Arheart, EdD, Department of Public Health Sciences, University of Miami Miller School of Medicine, Miami, FL, USA; Stephen L. Buka, SCD, Department of Epidemiology, Brown School of Public Health, Providence, Rl, USA

Correspondence: Professor Benjamin Vicente. Email: bvicent@udec.cl

First received 31 Jul 2019, final revision 27 Mar 2020, accepted 17 Apr 2020

\section{Supplementary material}

Supplementary material is available online at http://doi.org/10.1192/bjp.2020.88.

\section{Data availability}

Authors have ongoing access to de-identified data

\section{Acknowledgements}

We thank those who participated in this study and our colleagues in Chile, who graciously shared their data with us.

\section{Author contributions}

B.V., S.S. and R.K. conceptualised the study and collected the data. C.A.F., B.V., S.S and K.C.K obtained funding to analyse the data. C.A.F. and K.L.A. undertook the statistical analysis. C.A.F. wrote the first draft of the manuscript. K.W.C. Wrote the second draft of the manuscript. S.L.B., R.K., K.L.A., B.D.L.M. and K.C.K. mentored C.A.F. and served on her dissertation committee. All authors contributed to the proofreading and final approval of the manuscript.

\section{Funding}

This work was supported by the National Institute of Mental Health (grant F31MH104000 to C.A.F. and grant 5T32MH017119-30 to K.C.K.) and FONDEF Chile (grants D021-1140 and 1110687 to B.V.).

\section{Declaration of interest}

None.

ICMJE forms are in the supplementary material, available online at https://doi.org/10.1192/ bjp.2020.88.

\section{References}

1 Meichenbaum D, Novaco R. Stress inoculation: a preventative approach. Issues Ment Health Nurs 1985; 7: 419-35.

2 Updegraff JA, Taylor SE. From vulnerability to growth: positive and negative effects of stressful life events. In Loss and Trauma: General and Close Relationship Perspectives (ed H Miller): 3-28. Brunner-Routledge, 2000.

3 Southwick SM, Litz BT, Charney D, Friedman MJ. Resilience and Mental Health: Challenges Across the Lifespan. Cambridge University Press, 2011.

4 Seery MD, Leo RJ, Lupien SP, Kondrak CL, Almonte JL. An upside to adversity? Moderate cumulative lifetime adversity is associated with resilient responses in the face of controlled stressors. Psychol Sci 2013; 24: 1181-9.

5 Hammen C. Stress and depression. Annu Rev Clin Psychol 2005; 1: 293-19.

6 Yehuda R. Risk and resilience in posttraumatic stress disorder. J Clin Psychiatry 2004; 65(suppl 1): 29-36.

7 Van der Kolk BA, McFarlane AC, Weisaeth L. Traumatic Stress: The Effects of Overwhelming Experience on Mind, Body, and Society. Guilford Press, 2006. 
8 Breslau N, Chilcoat HD, Kessler RC, Davis GC. Previous exposure to trauma and PTSD effects of subsequent trauma: results from the Detroit Area Survey of Trauma. Am J Psychiatry 1999; 156: 902-7.

9 Farb NA, Irving JA, Anderson AK, Segal ZV. A two-factor model of relapse/ recurrence vulnerability in unipolar depression. J Abnorm Psychol 2015; 124 $38-53$

10 Monroe SM, Harkness KL. Life stress, the "kindling" hypothesis, and the recurrence of depression: considerations from a life stress perspective. Psychol Rev 2005; 112: 417-45.

11 Breslau N, Peterson EL, Schultz LR. A second look at prior trauma and the posttraumatic stress disorder effects of subsequent trauma: a prospective epidemiological study. Arch Gen Psychiatry 2008; 65: 431-7

12 Cougle JR, Resnick H, Kilpatrick DG. Does prior exposure to interpersonal violence increase risk of PTSD following subsequent exposure? Behav Res Ther 2009; 47: 1012-7.

13 Santos R, Byrnes B, Lane P. More than 2 million affected by earthquake, Chile's president says. CNN World, 28 Feb 2010. Available from: http://www.cnn.com/ 2010/WORLD/americas/02/27/chile.quake/.

14 Norris FH, Galea S, Friedman MJ, Watson PJ. Methods for Disaster Mental Health Research. Guilford Press, 2006

15 King M, Weich S, Torres-Gonzalez F, Svab I, Maaroos HI, Neeleman J, et al. Prediction of depression in European general practice attendees: the PREDICT study. BMC Public Health 2006; 6 : 6

16 King M, Walker C, Levy G, Bottomley C, Royston P, Weich S, et al. Development and validation of an international risk prediction algorithm for episodes of major depression in general practice attendees: the PredictD study. Arch Gen Psychiatry 2008; 65: 1368-76.

17 Bottomley C, Nazareth I, Torres-González F، Švab I, Maaroos HI, Geerlings MI et al. Comparison of risk factors for the onset and maintenance of depression. Br J Psychiatry 2010; 196: 13-7

18 Fernandez CA, Vicente B, Marshall B, Koenen KC, Arheart KL, Kohn R, et al. Longitudinal course of disaster-related PTSD among a prospective sample of adult Chilean natural disaster survivors. Int J Epidemiol 2017: 46: 440-52.

19 World Health Organization. Composite International Diagnostic Interview (CIDI, Version 2.1). WHO, 1997.

20 Robins LN, Wing J, Wittchen HU, Helzer JE, Babor TF, Burke J, et al. The Composite International Diagnostic Interview: an epidemiologic instrument suitable for use in conjunction with different diagnostic systems and in different cultures. Arch Gen Psychiatry 1988; 45: 1069-77.

21 Andrews G, Peters L. The psychometric properties of the Composite International Diagnostic Interview. Soc Psychiatry Psychiatr Epidemiol 1998; 33: $80-8$

22 Kessler RC, Ustun TB. The World Mental Health (WMH) Survey Initiative Version of the World Health Organization (WHO) Composite International Diagnostic Interview (CIDI). Int J Methods Psychiatr Res 2004; 13: 93-121.

23 Vicente B, Kohn R, Rioseco P, Saldivia S, Levav I, Torres S. Lifetime and 12month prevalence of DSM-III-R disorders in the Chile psychiatric prevalence study. Am J Psychiatry 2006; 163: 1362-70.

24 Vicente B, Kohn R, Rioseco P, Saldivia S, Navarrette G, Veloso P, et al. Regional differences in psychiatric disorders in Chile. Soc Psychiatry Psychiatr Epidemiol 2006; 41: 935-42.

25 Vielma M, Vicente B, Rioseco P, Castro P, Castro N, Torres S. Validación en Chile de la entrevista diagnóstica estandarizada para estudios epidemiológicos CIDI. Rev Psiquiatr 1992; 9: 1039-49.

26 American Psychiatric Association. Diagnostic and Statistical Manual of Mental Disorders (4th edn text revision) (DSM-IV-TR). APA, 2000

27 Brugha TS, Cragg D. The List of Threatening Experiences: the reliability and validity of a brief life events questionnaire. Acta Psychiatr Scand 1990; 82: 77-81.
28 Michalak EE, Tam EM, Manjunath CV, Yatham LN, Levitt AJ, Levitan RD, et al. Hard times and good friends: negative life events and social support in patients with seasonal and nonseasonal depression. Can J Psychiatry 2004; 49 408-11.

29 Friedman MJ, Keane TM, Resick PA. Handbook of PTSD: Science and Practice. Guilford Press, 2014

30 Brewin CR, Andrews B, Valentine JD. Meta-analysis of risk factors for posttraumatic stress disorder in trauma-exposed adults. J Consult Clin Psychol 2000; 68: 748-66.

31 Ozer EJ, Best SR, Lipsey TL, Weiss DS. Predictors of posttraumatic stress disorder and symptoms in adults: a meta-analysis. Psychol Bull 2003; 129: 52-73.

32 Robins JM, Hernan MA, Brumback B. Marginal structural models and causal inference in epidemiology. Epidemiology 2000; 11: 550-60.

33 Cole SR, Hernan MA. Constructing inverse probability weights for marginal structural models. Am J Epidemiol 2008; 168: 656-64.

34 StataCorp. Stata Statistical Software: Release 14. College Station, TX. StataCorp LP, 2015

35 Dienes KA, Hammen C, Henry RM, Cohen AN, Daley SE. The stress sensitization hypothesis: understanding the course of bipolar disorder. J Affect Disord 2006; 95: 43-9.

36 Nurius PS, Uehara E, Zatzick DF. Intersection of stress, social disadvantage, and life course processes: reframing trauma and mental health. Am J Psychiatr Rehabil 2013; 16: 91-114.

37 McLaughlin KA, Conron KJ, Koenen KC, Gilman SE. Childhood adversity, adult stressful life events, and risk of past-year psychiatric disorder: a test of the stress sensitization hypothesis in a population-based sample of adults Psychol Med 2010; 40: 1647-58

38 Harkness KL, Hayden EP, Lopez-Duran NL. Stress sensitivity and stress sensitization in psychopathology: an introduction to the special section. J Abnorm Psychol 2015; 124: 1-3.

39 Hankin BL, Badanes LS, Smolen A, Young JF. Cortisol reactivity to stress among youth: stability over time and genetic variants for stress sensitivity. J Abnorm Psychol 2015; 124: 54-67.

40 Karam EG, Friedman MJ, Hill ED, Kessler RC, McLaughlin KA, Petukhova M, et al. Cumulative traumas and risk thresholds: 12-month PTSD in the World Mental Health (WMH) surveys. Depress Anxiety 2014; 31: 130-42.

41 Miller KE, Omidian P, Rasmussen A, Yaqubi A, Daudzai H. Daily stressors, war experiences, and mental health in Afghanistan. Transcult Psychiatry 2008; 45 : 611-38

42 Newnham EA, Pearson RM, Stein A, Betancourt TS. Youth mental health after civil war: the importance of daily stressors. Br J Psychiatry 2015; 206: 116-21.

43 Kubiak SP. Trauma and cumulative adversity in women of a disadvantaged social location. Am J Orthopsychiatry 2005; 75: 451-65.

44 Ruscio AM, Gentes EL, Jones JD, Hallion LS, Coleman ES, Swendsen J. Rumination predicts heightened responding to stressful life events in major depressive disorder and generalized anxiety disorder. J Abnorm Psychol 2015; 124: $17-26$.

45 Fernandez CA, Loucks E, Arheart K, Hickson D, Kohn R, Buka SL, et al. Evaluating the effects of coping, social support and optimism on components of Allostatic Load - The Jackson Heart Study. Prev Chronic Dis 2015; 12: E165.

46 Monroe SM. Modern approaches to conceptualizing and measuring human life stress. Annu Rev Clin Psychol 2008; 4: 33-52. 